\title{
A Tripled fixed point theorem in partially ordered complete S-metric space
}

\author{
Mohammed Sani Mashina \\ Department of Mathematics Ahmadu Bello University, Zaria, Nigeria. \\ mohsanmash@gmail.com
}

Keywords: Partially ordered set, mixed monotone property, complete S-metric space, tripled fixed point.

\begin{abstract}
Sedghi et al. (Mat. Vesn. 64(3):258-266, 2012) introduced the notion of an $S$-metric as a generalized metric in 3-tuples $S: X^{3} \rightarrow[0, \infty)$, where $X$ is a nonempty set. In this paper we prove a tripled fixed point theorem for mapping having the mixed monotone property in partially ordered S-metric space. Our result generalize the result of Savitri and Nawneet Hooda (Int. J. Pure Appl. Sci. Technol. 20(1):111-116, 2014, On tripled fixed point theorem in partially ordered metric space) into the settings of $S$-metric space.
\end{abstract}

\section{Introduction}

Fixed point theory is an exciting branch of mathematics. It is a mixture of analysis, topology and geometry. The space $X$ is said to have the fixed point property for a map $T: X \rightarrow X$ if there exist $x \in X$ such that $T x=x$. Over the last 50 years or so, the theory of fixed point has been revealed as a very important tool in the study of nonlinear phenomena.

Ran and Reurings [11], Bhaskar and Lakshmikantham [1], Lakshmikantham and Ciric [6], Neito and Lopez [10], Mehta and Joshi [7], Berinde and Borcut [2] and Savitri and Hooda [12] proved some well-known results in partially ordered metric space. Berinde and Borcut [2] introduced the notion of tripled fixed point and proved some tripled fixed point theorem in partially ordered metric space.

Because modification, enrichment and extension of domain to a more general space is one of the active research in fixed point, some authors have tried to give generalization of metric space in several ways.

In 1963, Gähler [5] introduced the notion of a 2-metric space as follows:

Definition 1.1 Let $X$ be a nonempty set. A function $d: X^{3} \rightarrow \mathbb{R}$ is said to be a 2-metric on $X$ if for all $x, y, z, a \in X$, the following condition hold:

(d1) For any distinct point $x, y \in X$ there exist $z \in X, d(x, y, z) \neq 0$.

(d2) $d(x, y, z)=0$ if any of the two elements of the set $\{x, y, z\}$ in $X$ are equal.

(d3) $d(x, y, z)=d(x, z, y)=d(y, x, z)=d(z, x, y)=d(y, z, x)=d(z, y, x)$.

(d4) $d(x, y, z) \leq d(x, y, a)+d(x, a, z)+d(a, y, z)$.

The pair $(X, d)$ is called a 2 -metric space.

In 1984, Dhage in his Ph.D thesis [3] identified condition (d2) as a weakness in Gahler's theory of 2-metric space. To overcome these problems, he then introduced in [4] the concept of a $D$-metric space.

Definition 1.2 Let $X$ be a nonempty set. A function $D: X^{3} \rightarrow \mathbb{R}$ is called a $D$-metric on $X$ if for all $x, y, z, a \in X$, the following conditions hold:

(D1) $D(x, y, z) \geq 0$ for all $x, y, z, a$ in $\mathrm{X}$ and $D(x, y, z)=0$ if and only if $x=y=z$.

(D2) $D(x, y, z)=D(x, z, y)=D(y, x, z)=D(z, x, y)=D(y, z, x)=D(z, y, x)$. 
(D3) $D(x, y, z) \leq D(x, y, a)+D(x, a, z)+D(a, y, z)$.

The pair $(X, D)$ is called a $D$-metric space.

Mustafa and Sims [9] introduced the notion of $G$-metric space and suggested an important generalization of metric space as follows.

Definition 1.3 Let $X$ be a nonempty set. A function $G: X^{3} \rightarrow \mathbb{R}^{+}$is called a $G$-metric on $X$ if it satisfies the following condition: For all $x, y, z, a \in X$,

(G1) $G(x, y, z)=0$ if and only if $x=y=z$.

(G2) $0 \leq G(x, y, y)$ with $x \neq y$.

(G3) $G(x, x, y) \leq G(x, y, z)$ with $z \neq y$.

(G4) $G(x, y, z)=G(x, z, y)=G(y, x, z)=G(z, x, y)=G(y, z, x)=G(z, y, x)$.

(G5) $G(x, y, z) \leq G(x, a, a)+G(a, y, z)$.

The pair $(X, G)$ is called a $G$-metric space.

Thereafter Mustafa et al [10] proved some fixed point theorems in $G$-metric space.

Sedghi et al [15] introduced the notion of a $D^{*}$-metric space as follows.

Definition 1.4 Let $X$ be a nonempty set. A function $D^{*}: X^{3} \rightarrow \mathbb{R}^{+}$is called a $D^{*}$-metric on $X$ if it satisfies the following condition: For all $x, y, z, a \in X$,

(D*1) $D^{*}(x, y, z) \geq 0$.

(D*2) $D^{*}(x, y, z)=0$ if and only if $x=y=z$.

(D*3) $D^{*}(x, y, z)=D^{*}(x, z, y)=D^{*}(y, x, z)=D^{*}(z, x, y)=D^{*}(y, z, x)=D^{*}(z, y, x)$.

(D*4) $D^{*}(x, y, z) \leq D^{*}(x, y, a)+D^{*}(a, z, z)$.

The $\operatorname{pair}\left(X, D^{*}\right)$ is called a $D^{*}$-metric space.

Sedghi et al [16] introduced a new generalized metric space called an $S$-metric space.

Definition 1.5 Let $X$ be a nonempty set. An $S$-metric on $X$ is a function $S: X^{3} \rightarrow[0, \infty)$ that satisfies the following conditions. For each $x, y, z, a \in X$. (S1) $S(x, y, z) \geq 0$. (S2) $S(x, y, z)=0$ if and only if $x=y=z$. (S3) $S(x, y, z) \leq S(x, x, a)+S(y, y, a)+S(z, z, a)$.

The pair $(X, S)$ is called an $S$-metric space.

Example 1.1 (See [16]). Let $\mathbb{R}$ be the real line. Then $S(x, y, z)=|x-z|+|y-z|$ for all $x, y, z \in \mathbb{R}$ is an $S$-metric on. This $S$-metric is called the usual $S$-metric on $\mathbb{R}$.

Example 1.2 (See [16]) Let $X=\mathbb{R}^{2}$ and $d$ an ordinary metric on $X$. Then $S(x, y, z)=d(x, y)+d(x, z)+d(y, z)$ is an $S$-metric on $X$.

Example 1.3 (See [16]) Let $X=\mathbb{R}^{n}$ and $\|$.$\| a norm on X$. Then $S(x, y, z)=\|y+z-2 x\|+\|y-z\|$ is an $S$-metric on $X$.

Definition 1.6 (See [15]) Let $(X, S)$ be a $S$-metric space.

(1) A sequence $\left\{x_{n}\right\}, x \in X$ converges to $x$ if and only if $S\left(x_{n}, x_{n}, x\right) \rightarrow 0$ as $n \rightarrow \infty$. That is for each $\epsilon>0$ there exist $n_{0} \in \mathbb{N}$ such that for all $n \geq n_{0}, S\left(x_{n}, x_{n}, x\right)<\epsilon$ and we denote this bylim $\lim _{n \rightarrow \infty} x_{n}=x$.

(2) A sequence $x_{n}$ is called a Cauchy sequence if for each $\epsilon>0$, there exist $n_{0} \in \mathbb{N} \in$ such that $S\left(x_{n}, x_{n}, x_{m}\right)<\epsilon$ for each $n, m \geq n_{0}$.

(3) The $S$-metric space $(X, S)$ is said to be complete if every Cauchy sequence converges. 
Lemma 1.1 (See [16]) Let $(X, S)$ be an $S$-metric space. Then we have

$$
S(x, x, y)=S(y, y, x) \text {. }
$$

Lemma 1.2 (See [16]) Let $(X, S)$ be an $S$-metric space. Then

$$
S(x, x, z) \leq 2 S(x, x, y)+S(y, y, z) .
$$

Lemma 1.3 (See [16]) Let $(X, S)$ be an $S$-metric space. If the sequence $\left\{x_{n}\right\} \in X$ converges to $x$, then $x$ is unique.

Lemma 1.4 (See [16]) Let $(X, S)$ be an $S$-metric space. If the sequence $\left\{x_{n}\right\} \in X$ converges to $x$, then $\left\{x_{n}\right\}$ is a Cauchy sequence.

Lemma 1.5 (See [16]) Let $(X, S)$ be a $S$-metric space. If there exist sequences $\left\{x_{n}\right\}$ and $\left\{y_{n}\right\}$ in $X$ such that $\lim _{n \rightarrow \infty} x_{n}=x$ and $\lim _{n \rightarrow \infty} y_{n}=y$, then $\lim _{n \rightarrow \infty} S\left(x_{n}, x_{n}, y_{n}\right)=S(x, x, y)$.

Definition 1.7 (See [2]) Let $(X, \leq)$ be a partially ordered set and $d$ be a metric on $X$ such that $(X, d)$ is a complete metric space. Consider on the product space $X \times X \times X$ with the following partial order: for $(x, y, z),(u, v, w) \in X \times X \times X$,

$$
(u, v, w) \leq(x, y, z) \Leftrightarrow x \geq u, y \leq v, z \geq w .
$$

Definition 1.8 (See [2]) An element $(x, y, z) \in X \times X \times X$ is called a tripled fixed point of $F: X \times X \times X \rightarrow X$ if $F(x, y, z)=x, \quad F(y, x, y)=y$ and $F(z, y, x)=z$.

Definition 1.9 (See [2]) Let $(X, \leq)$ be a partially ordered set and $F: X \times X \times X \rightarrow X$. We say that $F$ has the mixed monotone property if $F(x, y, z)$ is monotone nondecreasing in $x$ and $z$ and is monotone non increasing in $y$, that is for any $x, y, z \in X$,

$$
\begin{aligned}
& x_{1}, x_{2} \in X, x_{1} \leq x_{2} \Rightarrow F\left(x_{1}, y, z\right) \leq F\left(x_{2}, y, z\right), \\
& y_{1}, y_{2} \in X, y_{1} \leq y_{2} \Rightarrow F\left(x, y_{1}, z\right) \geq F\left(x, y_{2}, z\right), \\
& z_{1}, z_{2} \in X, z_{1} \leq z_{2} \Rightarrow F\left(x, y, z_{1}\right) \leq F\left(x, y, z_{2}\right) .
\end{aligned}
$$

Theorem 1.1 (See [13]) Let $(X, \leq)$ be a partially ordered complete metric space and $F: X^{3} \rightarrow X$ be a continuous mapping having the mixed monotone property on $X$ and let there exists points $x_{0}, y_{0}, z_{0}$ with

$$
x_{0} \leq F\left(x_{0}, y_{0}, z_{0}\right), y_{0} \geq F\left(y_{0}, x_{0}, y_{0}\right), z_{0} \leq F\left(z_{0}, y_{0}, x_{0}\right) .
$$

Suppose that there exist non-negative real numbers $p$ and $q$ with $p+q<1$ such that

$$
\begin{aligned}
d(F(x, y, z), F(u, v, w)) \leq & p \min \{d(F(x, y, z), x), d(F(u, v, w), x)\} \\
& +q \min \{d(F(x, y, z), u), d(F(u, v, w), u)\}
\end{aligned}
$$

for all $x, y, z, u, v, w \in X$ with $x \geq u, y \leq v, z \geq w$. Then $F$ has a tripled fixed point in $X$.

The main aim of this paper is to generalize the result of Savitri and Hooda [12] into the structure of $S$-metric space.

\section{Main Results}

Theorem 2.1 Let $(X, \leq)$ be a partially ordered complete $S$-metric space and $F: X^{3} \rightarrow X$ be a continuous mapping having the mixed monotone property on $X$ and let there exists points $x_{0}, y_{0}, z_{0}$ with

$$
x_{0} \leq F\left(x_{0}, y_{0}, z_{0}\right), y_{0} \geq F\left(y_{0}, x_{0}, y_{0}\right), z_{0} \leq F\left(z_{0}, y_{0}, x_{0}\right)
$$


Suppose that there exist non-negative real numbers $\alpha$ and $\beta$ with $\alpha+\beta<1$ such that

$$
\begin{aligned}
& S(F(x, y, z), F(x, y, z), F(u, v, w)) \\
& \leq \alpha \min \{S(F(x, y, z), F(x, y, z), x), S(F(u, v, w), F(u, v, w), x)\} \\
& \quad+\beta \min \{S(F(x, y, z), F(x, y, z), u), S(F(u, v, w), F(u, v, w), u)\}
\end{aligned}
$$

for all $x, y, z, u, v, w \in X$ with $x \geq u, y \leq v, z \geq w$. Then $F$ has a tripled fixed point in $X$.

Proof. Let $x_{0}, y_{0}, z_{0} \in X$ with

$$
x_{0} \leq F\left(x_{0}, y_{0}, z_{0}\right), y_{0} \geq F\left(y_{0}, x_{0}, y_{0}\right), z_{0} \leq F\left(z_{0}, y_{0}, x_{0}\right)
$$

Define the sequences $\left\{x_{n}\right\},\left\{y_{n}\right\},\left\{z_{n}\right\}$ in $X$ such that for all $n=0,1,2, \ldots$

$x_{n+1}=F\left(x_{n}, y_{n}, z_{n}\right), \quad y_{n+1}=F\left(y_{n}, x_{n}, y_{n}\right), \quad z_{n+1}=F\left(z_{n}, y_{n}, x_{n}\right)$.

We claim that $\left\{x_{n}\right\},\left\{z_{n}\right\}$ are non-decreasing and $\left\{y_{n}\right\}$ is non-increasing. That is $n=0,1,2, \ldots$

$$
x_{n} \leq x_{n+1}, y_{n} \geq y_{n+1}, z_{n} \leq z_{n+1} \text {. }
$$

From (2) and (3), we have for $n=0$,

$$
\begin{aligned}
& x_{0} \leq F\left(x_{0}, y_{0}, z_{0}\right), y_{0} \geq F\left(y_{0}, x_{0}, y_{0}\right), z_{0} \leq F\left(z_{0}, y_{0}, x_{0}\right), \\
& x_{n+1}=F\left(x_{n}, y_{n}, z_{n}\right), \quad y_{n+1}=F\left(y_{n}, x_{n}, y_{n}\right), \quad z_{n+1}=F\left(z_{n}, y_{n}, x_{n}\right)
\end{aligned}
$$

This implies $x_{0} \leq x_{1}, y_{0} \geq y_{1}, z_{0} \leq z_{1}$.

Thus equation (4) holds for $n=0$.

Also suppose that (4) holds for some $n \in \mathbb{N}$. We now show that (4) is true for $n+1$.

Then by the mixed monotone property of $F$, we have

$$
\begin{aligned}
& x_{n+2}=F\left(x_{n+1}, y_{n+1}, z_{n+1}\right) \geq F\left(x_{n}, y_{n+1}, z_{n+1}\right) \geq F\left(x_{n}, y_{n}, z_{n+1}\right) \geq F\left(x_{n}, y_{n}, z_{n}\right), \\
& y_{n+2}=F\left(y_{n+1}, x_{n+1}, y_{n+1}\right) \leq F\left(y_{n}, x_{n+1}, y_{n+1}\right) \leq F\left(y_{n}, x_{n}, y_{n+1}\right) \leq F\left(y_{n}, x_{n}, y_{n}\right), \\
& z_{n+2}=F\left(z_{n+1}, y_{n+1}, x_{n+1}\right) \geq F\left(z_{n}, y_{n+1}, x_{n+1}\right) \geq F\left(z_{n}, y_{n}, x_{n+1}\right) \geq F\left(z_{n}, y_{n}, x_{n}\right) .
\end{aligned}
$$

Thus by mathematical induction, equation (4) holds for $n \in \mathbb{N}$.

Therefore,

$$
\begin{aligned}
& x_{0} \leq x_{1} \leq x_{2} \leq \ldots \leq x_{n} \leq x_{n+1} \ldots, \\
& y_{0} \geq y_{1} \geq y_{2} \geq \ldots \geq y_{n} \geq y_{n+1} \ldots, \\
& z_{0} \leq z_{1} \leq z_{2} \leq \ldots \leq z_{n} \leq z_{n+1} \ldots .
\end{aligned}
$$

As $x_{n} \geq x_{n-1}, y_{n} \leq y_{n-1}, z_{n} \geq z_{n-1}$, we have from (1)

$S\left(F\left(x_{n}, y_{n}, z_{n}\right), F\left(x_{n}, y_{n}, z_{n}\right), F\left(x_{n-1}, y_{n-1}, z_{n-1}\right)\right)$

$\leq \alpha \min \left\{S\left(F\left(x_{n}, y_{n}, z_{n}\right), F\left(x_{n}, y_{n}, z_{n}\right), x_{n}\right), S\left(F\left(x_{n-1}, y_{n-1}, z_{n-1}\right), F\left(x_{n-1}, y_{n-1}, z_{n-1}\right), x_{n}\right)\right\}$

$+\beta \min \left\{S\left(F\left(x_{n}, y_{n}, z_{n}\right), F\left(x_{n}, y_{n}, z_{n}\right), x_{n-1}\right), S\left(F\left(x_{n-1}, y_{n-1}, z_{n-1}\right), F\left(x_{n-1}, y_{n-1}, z_{n-1}\right), x_{n-1}\right)\right\}$

$=\alpha \min \left\{S\left(x_{n+1}, x_{n+1}, x_{n}\right), S\left(x_{n}, x_{n}, x_{n}\right)\right\}+\beta \min \left\{S\left(x_{n+1}, x_{n+1}, x_{n-1}\right), S\left(x_{n}, x_{n}, x_{n-1}\right)\right.$

$=\beta S\left(x_{n}, x_{n}, x_{n-1}\right)$.

Hence $S\left(x_{n+1}, x_{n+1}, x_{n}\right) \leq \beta S\left(x_{n}, x_{n}, x_{n-1}\right)$.

Again as $y_{n} \leq y_{n-1}, x_{n} \geq x_{n-1}$, we have from (1)

$S\left(F\left(y_{n-1}, x_{n-1}, y_{n-1}\right), F\left(y_{n-1}, x_{n-1}, y_{n-1}\right), F\left(y_{n}, x_{n}, y_{n}\right)\right)$

$\leq \alpha \min \left\{S\left(F\left(y_{n-1}, x_{n-1}, y_{n-1}\right), F\left(y_{n-1}, x_{n-1}, y_{n-1}\right), y_{n-1}\right), S\left(F\left(y_{n}, x_{n}, y_{n}\right), F\left(y_{n}, x_{n}, y_{n}\right), y_{n-1}\right)\right\}$

$+\beta \min \left\{S\left(F\left(y_{n-1}, x_{n-1}, y_{n-1}\right), F\left(y_{n-1}, x_{n-1}, y_{n-1}\right), y_{n}\right), S\left(F\left(y_{n}, x_{n}, y_{n}\right), F\left(y_{n}, x_{n}, y_{n}\right), y_{n}\right)\right\}$

$=\alpha \min \left\{S\left(y_{n}, y_{n}, y_{n-1}\right), S\left(y_{n+1}, y_{n+1}, y_{n-1}\right)\right\}+\beta \min \left\{S\left(y_{n}, y_{n}, y_{n}\right), S\left(y_{n+1}, y_{n+1}, y_{n}\right)\right.$

$=\alpha S\left(y_{n}, y_{n}, y_{n-1}\right)$.

Hence $S\left(y_{n+1}, y_{n+1}, y_{n-1}\right) \leq \alpha S\left(y_{n}, y_{n}, y_{n-1}\right)$.

Finally as $z_{n} \geq z_{n-1}, y_{n} \leq y_{n-1}, x_{n} \geq x_{n-1}$, we have from (1) 
$S\left(F\left(z_{n}, y_{n}, x_{n}\right), F\left(z_{n}, y_{n}, x_{n}\right), F\left(z_{n-1}, y_{n-1}, x_{n-1}\right)\right)$

$\leq \alpha \min \left\{S\left(F\left(z_{n}, y_{n}, x_{n}\right), F\left(z_{n}, y_{n}, x_{n}\right), z_{n}\right), S\left(F\left(z_{n-1}, y_{n-1}, x_{n-1}\right), F\left(z_{n-1}, y_{n-1}, x_{n-1}\right), z_{n}\right)\right\}$

$+\beta \min \left\{S\left(F\left(z_{n}, y_{n}, x_{n}\right), F\left(z_{n}, y_{n}, x_{n}\right), z_{n-1}\right), S\left(F\left(z_{n-1}, y_{n-1}, x_{n-1}\right), F\left(z_{n-1}, y_{n-1}, x_{n-1}\right), z_{n-1}\right)\right\}$

$=\alpha \min \left\{S\left(z_{n+1}, z_{n+1}, z_{n}\right), S\left(z_{n}, z_{n}, z_{n}\right)\right\}+\beta \min \left\{S\left(z_{n+1}, z_{n+1}, z_{n-1}\right), S\left(z_{n}, z_{n}, z_{n-1}\right)\right.$

$=\beta S\left(z_{n}, z_{n}, z_{n-1}\right)$.

Hence $S\left(z_{n+1}, z_{n+1}, z_{n}\right) \leq \beta S\left(z_{n}, z_{n}, z_{n-1}\right)$.

Adding (5), (6) and (7), we get

$$
\begin{aligned}
& S\left(x_{n+1}, x_{n+1}, x_{n}\right)+S\left(y_{n+1}, y_{n+1}, y_{n-1}\right)+\left(z_{n+1}, z_{n+1}, z_{n}\right) \\
& \leq \beta S\left(x_{n}, x_{n}, x_{n-1}\right)+\alpha S\left(y_{n}, y_{n}, y_{n-1}\right)+\beta S\left(z_{n}, z_{n}, z_{n-1}\right) \\
& =\beta\left[S\left(x_{n}, x_{n}, x_{n-1}\right)+S\left(z_{n}, z_{n}, z_{n-1}\right)\right]+\alpha S\left(y_{n}, y_{n}, y_{n-1}\right) \\
& \leq(\alpha+\beta)\left[S\left(x_{n}, x_{n}, x_{n-1}\right)+S\left(z_{n}, z_{n}, z_{n-1}\right)\right]+(\alpha+\beta) S\left(y_{n}, y_{n}, y_{n-1}\right) \\
& =(\alpha+\beta)\left[S\left(x_{n}, x_{n}, x_{n-1}\right)+S\left(y_{n}, y_{n}, y_{n-1}\right)+S\left(z_{n}, z_{n}, z_{n-1}\right)\right] .
\end{aligned}
$$

Let $A=\alpha+\beta<1$. Then

$$
\begin{aligned}
& S\left(x_{n+1}, x_{n+1}, x_{n}\right)+S\left(y_{n+1}, y_{n+1}, y_{n-1}\right)+\left(z_{n+1}, z_{n+1}, z_{n}\right) \\
& \leq A\left[S\left(x_{n}, x_{n}, x_{n-1}\right)+S\left(y_{n}, y_{n}, y_{n-1}\right)+S\left(z_{n}, z_{n}, z_{n-1}\right)\right] \\
& \leq A^{2}\left[S\left(x_{n-1}, x_{n-1}, x_{n-2}\right)+S\left(y_{n-1}, y_{n-1}, y_{n-2}\right)+S\left(z_{n-1}, z_{n-1}, z_{n-2}\right)\right. \\
& \cdot \\
& \cdot \\
& \leq \\
& \leq A^{n}\left[S\left(x_{1}, x_{1}, x_{0}\right)+S\left(y_{1}, y_{1}, y_{0}\right)+S\left(z_{1}, z_{1}, z_{0}\right)\right] .
\end{aligned}
$$

Moreover by lemma 1.2, we have for all $n \leq m$

$$
\begin{aligned}
& S\left(x_{n}, x_{n}, x_{m}\right)+S\left(y_{n}, y_{n}, y_{m}\right)+S\left(z_{n}, z_{n}, z_{m}\right) \\
& \leq\left(2 S\left(x_{n}, x_{n}, x_{n+1}\right)+2 S\left(y_{n}, y_{n}, y_{n+1}\right)+2 S\left(z_{n}, z_{n}, z_{n+1}\right)\right) \\
& +\left(S\left(x_{n+1}, x_{n+1}, x_{n}\right)+S\left(y_{n+1}, y_{n+1}, y_{n-1}\right)+S\left(z_{n+1}, z_{n+1}, z_{n}\right)\right) \\
& \leq\left(2 S\left(x_{n}, x_{n}, x_{n+1}\right)+2 S\left(y_{n}, y_{n}, y_{n+1}\right)+2 S\left(z_{n}, z_{n}, z_{n+1}\right)\right) \\
& +\left(2 S\left(x_{n+1}, x_{n+1}, x_{n}\right)+2 S\left(y_{n+1}, y_{n+1}, y_{n-1}\right)+2 S\left(z_{n+1}, z_{n+1}, z_{n}\right)\right) \\
& +\ldots+\left(2 S\left(x_{m-2}, x_{m-2}, x_{m-1}\right)+2 S\left(y_{m-2}, y_{m-2}, y_{m-1}\right)+2 S\left(z_{m-2}, z_{m-2}, z_{m-1}\right)\right) \\
& +\left(S\left(x_{m-1}, x_{m-1}, x_{m}\right)+S\left(y_{m-1}, y_{m-1}, y_{m}\right)+S\left(z_{m-1}, z_{m-1}, z_{m}\right)\right) \\
& \leq\left(2 S\left(x_{n}, x_{n}, x_{n+1}\right)+2 S\left(y_{n}, y_{n}, y_{n+1}\right)+2 S\left(z_{n}, z_{n}, z_{n+1}\right)\right) \\
& +\ldots+\left(2 S\left(x_{m-1}, x_{m-1}, x_{m}\right)+2 S\left(y_{m-1}, y_{m-1}, y_{m}\right)+2 S\left(z_{m-1}, z_{m-1}, z_{m}\right)\right) \\
& \leq 2\left[\left(A^{n}+A^{n+1}+\ldots+A^{m-1}\right)\left(S\left(x_{0}, x_{0}, x_{1}\right)+S\left(y_{0}, y_{0}, y_{1}\right)+S\left(z_{0}, z_{0}, z_{1}\right)\right)\right] \\
& \leq \frac{2 A^{n}}{1-A}\left(S\left(x_{0}, x_{0}, x_{1}\right)+S\left(y_{0}, y_{0}, y_{1}\right)+S\left(z_{0}, z_{0}, z_{1}\right)\right) .
\end{aligned}
$$

Since $A<1$, taking limit as $n, m \rightarrow \infty$, we get

$\lim _{n, m \rightarrow \infty}\left\{S\left(x_{n}, x_{n}, x_{m}\right)+S\left(y_{n}, y_{n}, y_{m}\right)+S\left(z_{n}, z_{n}, z_{m}\right)\right\}=0$.

This implies that

$\lim _{n, m \rightarrow \infty} S\left(x_{n}, x_{n}, x_{m}\right)=\lim _{n, m \rightarrow \infty} S\left(y_{n}, y_{n}, y_{m}\right)=\lim _{n, m \rightarrow \infty} S\left(z_{n}, z_{n}, z_{m}\right)=0$.

Therefore $\left\{x_{n}\right\},\left\{y_{n}\right\}$ and $\left\{z_{n}\right\}$ are Cauchy sequences in $X$. 
Since $X$ is complete, there exist $x, y, z \in X$ such that as $n \rightarrow \infty, x_{n} \rightarrow x, y_{n} \rightarrow y$ and $z_{n} \rightarrow z$. Hence by taking limit as $n \rightarrow \infty$ in (3), we get

$$
\begin{aligned}
& x=\lim _{n \rightarrow \infty} x_{n}=\lim _{n \rightarrow \infty} F\left(x_{n-1}, y_{n-1 .} z_{n-1}\right)=F\left(\lim _{n \rightarrow \infty} x_{n-1} \cdot \lim _{n \rightarrow \infty} y_{n-1} \cdot \lim _{n \rightarrow \infty} z_{n-1}\right)=F(x, y, z), \\
& y=\lim _{n \rightarrow \infty} y_{n}=\lim _{n \rightarrow \infty} F\left(y_{n-1}, x_{n-1} . y_{n-1}\right)=F\left(\lim _{n \rightarrow \infty} y_{n-1} \lim _{n \rightarrow \infty} x_{n-1} \cdot \lim _{n \rightarrow \infty} y_{n-1}\right)=F(y, x, y), \\
& z=\lim _{n \rightarrow \infty} z_{n}=\lim _{n \rightarrow \infty} F\left(z_{n-1}, y_{n-1 .} x_{n-1 .}\right)=F\left(\lim _{n \rightarrow \infty} z_{n-1} \cdot \lim _{n \rightarrow \infty} y_{n-1} \cdot \lim _{n \rightarrow \infty} x_{n-1}\right)=F(z, y, x) .
\end{aligned}
$$

Thus we have $F(x, y, z)=x, F(y, x, y)=y, F(z, y, x)=z$ and $F$ has a tripled fixed point

We now give an example to support our result.

Example 2.1 Let $X=[0,1] \quad x \leq y \Leftrightarrow x, y \in[0,1]$ with the usual order $\leq$. Let $(X, \leq, S)$ be a partially ordered complete $S$-metric with the usual $S$-metric defined as in example 1.1. That is $S(x, y, z)=|x-z|+|y-z|$. So, $S(x, x, y)=|x-y|+|x-y|=2|x-y|=2 d(x, y)$.

Define $F: X \times X \times X \rightarrow X$ by

$$
F(x, y, z)=\left\{\begin{array}{cc}
\frac{x-y}{8}, & \text { if } x \geq y \\
\frac{y-z}{8}, & \text { if } y \geq z \\
\frac{z-x}{8}, & \text { if } z \geq x \\
\frac{1}{8}, & \text { otherwise }
\end{array}\right.
$$

Then $F$ is continuous and has the mixed monotone property.

Let there exist $x_{0}=y_{0}=z_{0}=0$ such that

$x_{0}=0 \leq F(0,0,0)=F\left(x_{0}, y_{0}, z_{0}\right), y_{0}=0 \geq F(0,0,0)=F\left(y_{0}, x_{0}, y_{0}\right)$ and $z_{0}=0 \leq F(0,0,0)=F\left(z_{0}, y_{0}, x_{0}\right)$.

Next we show that the mapping $F$ satisfies (1) with $\alpha=\beta=\frac{1}{8}$

For $x, y, z, u, v, w \in X, x \geq u, y \leq v, z \geq w$ such that the following eight cases hold.
1. $x=u, y<v, z=w$.
2. $x=u, y<v, z>w$.
3. $x=u, y=v, z=w$.
4. $x=u, y<v, z>w$.
5. $x>u, y<v, z=w$.
6. $x>u, y<v, z>w$.
7. $x>u, y=v, z>w$.
8. $x>u, y=v, z=w$.

If $(x, y, z)=(0,0,1)$ and $(u, v, w)=(0,1,1)$, we consider the possibility of case 1 for the maximum and minimum values of $x, y, z, u, v, w \in X$ using (1).

It is clear that L.H.S. of (1) is 0. i.e.

$$
S(F(x, y, z), F(x, y, z), F(u, v, w))=2 d(F(x, y, z), F(u, v, w))=0 .
$$


R.H.S of (1) is given by

$\frac{1}{8} \min \{S(F(x, y, z), F(x, y, z), x), S(F(u, v, w), F(u, v, w), x)\}$

$+\frac{1}{8} \min \{S(F(x, y, z), F(x, y, z), u), S(F(u, v, w), F(u, v, w), u)\}$

$=\frac{1}{8} \min \{2 d(F(x, y, z), x), 2 d(F(u, v, w), x)\}+\frac{1}{8} \min \{2 d(F(x, y, z), u), 2 d(F(u, v, w), u)\}$

$=\frac{1}{8} \min \left\{2\left(\frac{1}{8}, 0\right), 2\left(\frac{1}{8}, 0\right)\right\}+\frac{1}{8} \min \left\{2\left(\frac{1}{8}, 0\right), 2\left(\frac{1}{8}, 0\right)\right\}=\frac{2}{32}=\frac{1}{16}$.

So (1) is satisfied and $F$ has a tripled fixed point.

\section{References}

[1] T.G. Bhaskar and V. Lakshmikantham, Fixed point theorems in partially ordered metric spaces and applications, Nonlinear Anal., 65(2006), 1379-1393.

[2] V. Berinde and M. Borcut, Tripled fixed point theorems for contractive type mappings in partially ordered metric spaces, Nonlinear Anal., 74(15) (2011), 4889-4897.

[3] B. C. Dhage, A study of some fixed point theorems, Ph.D. Thesis, Marathwada Univ. Aurangabad, India 1984.

[4] B. C. Dhage, Generalized metric space and mappings with fixed point, Bull.Cal.Math. Soc., 84 (1992) $329-336$.

[5] S. Gähler, 2-metriche raume und ihre toplogische strukture. Maths.Nachr. , 26 (1963) 115-148.

[6] P. Kumar and A. Poonam, Fixed point theorem for $\alpha-\psi$-contractive type mappings in 2metric space, Inter.J. math.Trend.Tech. 10(1), (2014) 34-37.

[7] V. Lakshmikantham and L. Ciric, Coupled fixed point theorems for nonlinear contractions in partially ordered metric spaces, Nonlinear Anal., 70(2009), 4341-4349.

[8] G.J. Mehta and M.L. Joshi, Coupled fixed point theorem in partially ordered complete metric space, Int. J. Pure Appl. Sci. Technol., 1(2) (2010), 87-96.

[9] Z. Mustafa and B. Sims, A new approach to generalize metric space, J. Roy.nonlinear convex anal. 7(2006) $289-297$.

[10]Z. Mustafa, H. Obiedat and F. Awawdeh, Some common fixed point theorems for mapping on complete $G$-metric space, Fixed Point Theory Appl.2008 (2008) Article ID 189870.

[11] J.J. Nieto and R.R. Lopez, Existence and uniqueness of fixed point in partially ordered sets and applications to ordinary differential equations, Acta Mats. Sinica (Engl. Ser.), 23(12) (2007), 2205-2212.

[12] A.C.M. Ran and M.C.B. Reurings, A fixed point theorem in partially ordered sets and some applications to metric equations, Proc. Amer. Math. Soc., 132(2004), 1435-1443.

[13] Savitri and N. Hooda, On tripled fixed point theorem in partially ordered complete metric space, Int. J. Pure Appl. Sci. Technol., 20(1) (2014), 111-116.

[14]B. Samet, C. Vetro and P. Vetro, Fixed point theorem for $\alpha-\psi$-contractive type mappings, Nonlinear Anal. 75(2012) 2154-2165.

[15] S. Sedghi, N. Shobe and H. Zhou, A common fixed point theorem in $D^{*}$-metric space, Fixed Point Theory Appl.2007 (2007) Article ID 027906.

[16] S. Sedghi, N. Shobe and A. Aliouche, A generalization of fixed point theorem in $S$-metric space, Mat.Vesinik. 64(3), (2012) 258 -266. 